\title{
Optical Absorption of Nano-Composite Thin Films of Au in Teflon
}

\author{
Dmytro Grynko $^{1}$, Konstantin Grytrsenko ${ }^{1}$, Valeri Lozovski ${ }^{1,2}$, Mykola Sopinskyy ${ }^{1}$, Galyna Strilchuk ${ }^{1,2}$ \\ ${ }^{1}$ V.Lashkaryov Institute of Semiconductor Physics, National Academy of Sciences of Ukraine, Kyiv, Ukraine; ${ }^{2}$ Kyiv T.Shevchenko \\ National University, Institute of High Technologies, Kyiv, Ukraine. \\ Email: gstrilchuk@gmail.com
}

Received May $13^{\text {th }}, 2010$; revised June $13^{\text {th }}, 2010$; accepted June $23^{\text {rd }}, 2010$.

\begin{abstract}
Nano-composite films of Au particles in Teflon were obtained by thermal vacuum deposition. The obtained films were characterized by the different shapes and dimensions of the inclusion particles. Absorption spectra of the films were measured in-situ. A model for the calculation of the optical properties of the nano-composite thin films with an inhomogeneous distribution of the inclusions along the thickness of the film is proposed. Absorption properties of inclusions were analyzed by considering the local field interaction. The calculated absorption profiles are compared with the experimentally obtained absorption profiles. This comparison gives a possibility to draw conclusions about the concentration, shapes and shape distributions of the inclusion particles. For example, the films obtained by duration deposition are characterized by inclusions having the shape of prolate ellipsoids oriented normally to surface of the film.
\end{abstract}

Keywords: Nano-Composite, Optical Absorption Spectra, Thin Film, Effective Susceptibility, Teflon, Gold, Vacuum Deposition

\section{Introduction}

The study of optical properties of thin nano-composite fi$1 \mathrm{~ms}$ is a powerful tool for understanding the physical properties and the spatial structure of the material. The tailoring of physical properties can be performed by choosing appropriate particle sizes, opening the way to a composite material with remarkable effects, especially in optoelectronics. The development of composite materials based on semiconductor quantum dots (see for example, [1]) is an example of these applications. Much attention has been focused on the physical and optical properties of metallic clusters embedded in a dielectric matrix. It is well known that metallic nanoparticles show clear size effects in their thermodynamic properties [1-4]. Nanoclusters, mostly of noble metals, are widely studied for applications in sensors [5-9], photonics devices and plasmonic structures [10], waveguides [11], films for photovoltaics [12], medicine [13], chemical catalysis [14], filters [15], electronic devices [16,17], etc. In this work we consider $\mathrm{Au} / \mathrm{Teflon}$ nano-composites.

The optical properties, such as absorption and luminescence [18], are widely used for the determination of changes in the structure of metal/polymer films [15], understanding of electrical processes in thin films, analysis of particles sizes [19], shapes [16] and sizes distributions
[16]. We shall analytically simulate the absorption spectra and thus have an opportunity to analyze the parameters of the film. These simulations can be performed by taking into account the inhomogeneity of the film and the presence of the third phase (air).

It is well known that the macroscopic Maxwell equations can be used only when the characteristic wavelength $\lambda$ of the system is much larger than the scale of the microfield inhomogeneities $l(\lambda \gg l)$, which can be comparable to the thicknesses of transition layers in the surface or interface domains. Also, when we deal with thin films we should take into account the near-interface layers at both sides. In the case of thin films where the nearinterface layer length is comparable to the thickness of the film, the local field inhomogeneity plays an essential role, which makes the use of microscopic approaches not expedient. Indeed, on one hand such an approach is mathematically complex and, on the other hand, it depends strongly on the specific model. It is much more reasonable to take advantage of a mesoscopic approach, which has been actively developed in near-field optics, in particular, in methods of scanning near-field optical microscopy [20-25]. Therefore, we shall consider the electrodynamics of thin and ultrathin films in the framework of the self-consistent (local) field approach, which does not directly involve the Maxwell boundary conditions, and ta- 
kes into account the effects related to the local field inhomogeneity. This approach is based on the Green's function method. One calculates absorption profiles via the dissipative function. It is quite adequate for solving the stated problem and, as was noted above, is often used in different areas of physics, for example, in near-field optics.

We shall develop a method which allows us to determine the parameters of nano-composite films, which can not be measured directly. The main idea of the approach consists of comparison of experimentally measured and theoretically calculated absorption profiles. The absorption spectrum sensibly depends on the film parameters. There is a metal concentration in a film, presence of pores, shells of particles and the thickness of this shell, distribution of particles along the thickness of the film, the shape distribution of particles (from oblate ellipsoids, spheres, up to prolate ellipsoids), and the orientation of particles in a spatial layer.

\section{Experimental Details}

There is a number of methods for the preparation of metal/insulator composite films [16-18,26-28], particularly for $\mathrm{Au} / \mathrm{Teflon}$ nano-composites. Our films were made by thermal vacuum deposition. The method is based on Teflon evaporation with vapor activation by an electron clo$\mathrm{ud}$, with an electron energy near $1 \mathrm{keV}$, and a plasma treatment has been proposed recently $[6,29]$.

$\mathrm{Au} / \mathrm{Teflon}$ nano-composites with a thickness less than $100 \mathrm{~nm}$ and with a metal volume fraction over the range $7 \%-85 \%$ were deposited using vacuum VUP-5 installation with a residual pressure of $10^{-3} \mathrm{~Pa}$. The original computerized system was used to control the film deposition process in-situ.

The control of the molecular beam of each nano-composite component and deposited film is realized by the quartz monitor technique with a sensitivity of $6 \times 10^{-8} \mathrm{~kg} /$ $\mathrm{m}^{2} \mathrm{~Hz}$. The optical spectrometer equipped with fibres ledin vacuum chamber gives much diverse spectroscopic information on film growth processes in-situ. The sample delivery system expands the set of sample operations at the time of film deposition and allows depositing uniform or wedge-like by thickness films when needed. Water-cooled walls of each evaporator shadow the thermal radiation and prevent samples from heating, which is significant for the condensation of volatile organic compounds. A computerized system carried out the process control. The advantages of the described co-deposition method are its universality, simplicity for choosing composites with different components, and the ability to control and to modify growth processes in-situ, in real time.

The block diagram of the installation for the threecomponent nano-composite films deposition in vacuum is shown in Figure 1. The deposition process takes place in vacuum chamber 1 (with a volume of $50 \mathrm{dm}^{3}$ ) of automatic control vacuum installation VUP-5. This method allows us to make a nanolayer structure which consists of Teflon and gold discontinuous layers.

The evaporation of nano-composites from $\mathrm{Au}$ and Teflon sources is controlled by varying the evaporation speed for each nano-composite component. The molecular beam density was obtained with quartz microbalances. The deposition rate was measured by the quartz microbalance 6 in the control measurement. Sample-to-source factors (which consider a solid angle, comparing the evaporation angle of gold (or Teflon) on the quartz microbalances and on a film plane) have been calculated by a frequency shift of the microbalance 6 and of the microbalance 8 (Figure 1) in the control measurement.

The metal mass concentration has been estimated by measuring the quartz frequency shifts during the process of deposition [30], taking into account sample-to-source factors and condensation factors. The volume fraction was determined using metal mass concentration and the densities of gold films and Teflon films $[31,32]$. The condensation factor (or sticking factor, which considers a difference between $\mathrm{Au}$ content in a beam from the source and $\mathrm{Au}$ condensed on the Teflon film) has been measured in the calibration experiment by direct registration of frequency shifts for the quartz fixed on the stationary shield and for the quartz rotated with the sample holder. The condensation factor has been calculated as 0.18 from the present data. The Teflon film density is calculated from the frequency shift of the quartz microbalances rotated with the sample holder. The film thickness was measured by atomic force microscope (AFM), using step.

Rotating glass disks with attached glass and KCI crystal slides were used as substrates. The film growth rate was about $7 \mathrm{~nm} / \mathrm{min}$, and the substrate rotation speed was

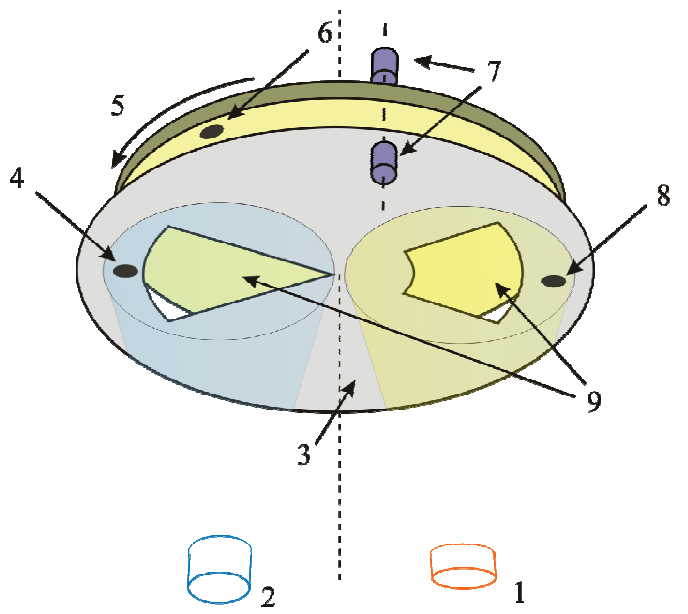

Figure 1. Scheme for Au-Teflon composites co-condensation vacuum setup: 1-Au evaporator, 2-Teflon evaporator , 3stationary protective shield, 4,8-stationary quartz microbalance fixed on shield, 5-rotating sample holder, 6-quartz microbalance fixed on rotating sample holder, 7-spectrophotometer fibers, 9-evaporation aperture 
about $50 \mathrm{~min}^{-1}$. To obtain direct evidences of the size and shape of the gold particles in the film, transmission electron microscopy (TEM) with measurements at different angles (normal and $60^{\circ}$ ) to the film plane was used (Figure 2). The images were analyzed with the help of twodimensional Fourier transforms [33]. This technique allowed us to determine the size distribution of the particles. The parameters of the size distribution in various lateral directions allowed us to determine the shape distribution of the particles and to analyze the structure of the composite. As indirect evidence for the size and the shape of the gold inclusions, we use simulated optical properties of the films. Both approaches for finding films structure are used.

Direct experimental investigation of the film surface can offer important information about the gold particles. Three samples were investigated, the TEM's of which (normal and $60^{\circ}$ incidence angle) are shown in Figure 2. The first sample, Au-1, (Figures 2(a) and 2(b)) has a gold mass concentration of $83 \%$ (volume fraction of $35 \%$ ). The thickness of the film is $26 \mathrm{~nm}$, the diameter of particles is $d=2.5-5 \mathrm{~nm}$. This thickness was obtained by AFM measurements. The TEM study has shown that the diameters of the particles in this sample in perpendicular and parallel directions to the film plane are approximately equal to each other. Therefore, one can assume the inclusion particles to be practically spherical. The shape of the particles in a nano-composite produced through a similar method was investigated elsewhere [15-18]. In most cases one can see from the TEM images that the particles are near-spherical. In our TEM images we also notice this, despite the fact that we do not see a single layer of nanoparticles. Two-angle images show that the particles have a similar form when viewed with a different projection. The particles may not be perfectly ellipsoidal, but we suppose that it can be used for modeling with some inaccuracy. This inaccuracy we take into account by using the distribution function of particle shape.

But a more complicated structure of the composite appears at higher concentrations of the inclusions. This situation is beyond our scope because this structure is characterized by nonellipsoidal shapes of the particles.

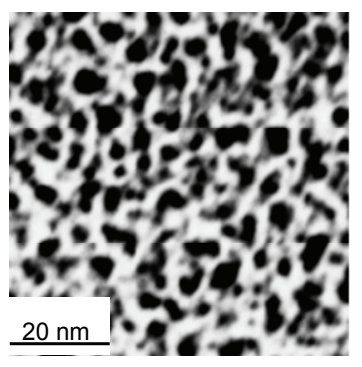

(a)

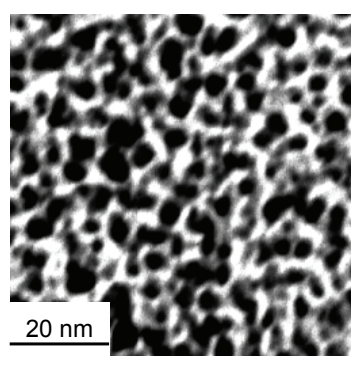

(b)
Figure 2. TEM images of Au/Teflon nano-composites for (a) $83 \%$, normal incidence; (b) $83 \%, 60^{\circ}$ angle of incidence
The Au-2 sample (TEM image shown in Figure 3(a) has a concentration less than the Au-1 sample and equals $56 \%$ (volume fraction of $15 \%$ ). The diameter of the particles in this case is $d=4-7 \mathrm{~nm}$. According to the microscopy data, particles in the film are prolate with the large axis perpendicular to the film plane. The Au-3 sample (TEM image shown in Figure 3(b)) has a $\mathrm{Au}$ mass concentration of $14 \%$ (volume fraction of $2 \%$ ). There is a non-porous, non-conductive film with a thickness of $27 \mathrm{~nm}$ and spherical particles with a diameter of $d$ $=1.4 \mathrm{~nm}$. TEM images show nearly the same size of particles in each film.

The in-situ measurement of absorption spectra gives us additional information about the optical properties and the structure of the film during its growth. Namely, the different absorption profiles obtained at some intervals of growth time reflect the temporal evolution of light absorption by the films. Since absorption properties are noticeably determined by the film structure, the temporal evolution of the absorption profile reflects the temporal evolution of the film structure. The technology regime, obviously, determines the geometrical structure of the composite films. These films have different optical properties. In Figure 4 the dependence of the light absorption

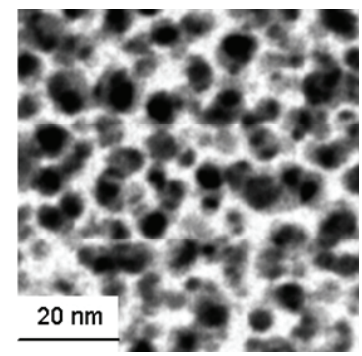

(a)

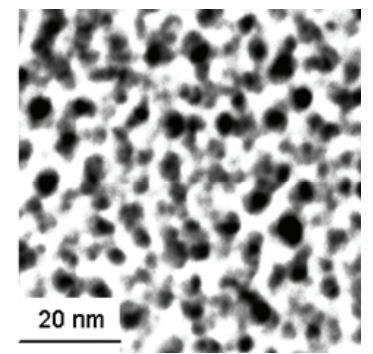

(b)
Figure 3. TEM sketch of Au/Teflon nano-composites for (a) $56 \%$; (b) $14 \%$ metal mass fraction normal incidence

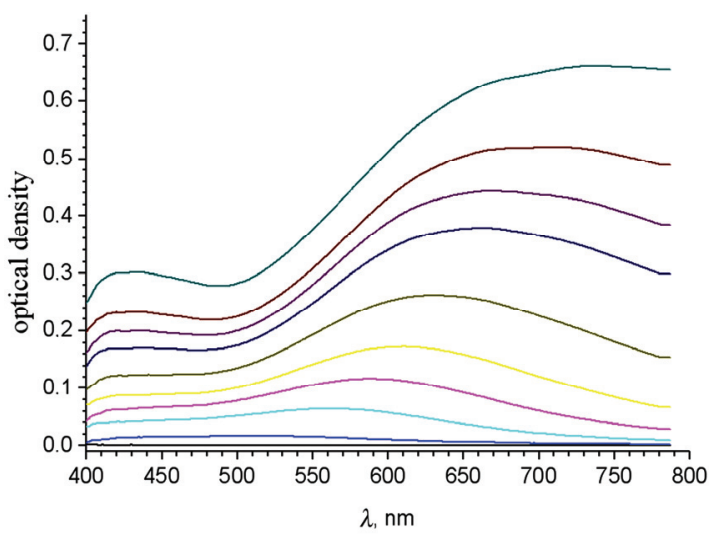

Figure 4. Absorption profiles measured during growth (bottom-up) of the film, for $35 \%$ volume concentration of gold in Teflon 
profiles on the growth time is represented for the Au-3 sample. The dynamics of the change in absorption profile gives us information about formation of gold particles in the process of growth. The absorption spectra are measured at equal intervals during the growth of the film Au-1. Displacement of an absorption spectrum maximum to red wavelengths testifies to the inhomogeneity of the film. Teflon is warmed up during the process of evaporation and gold sticking coefficient varies. For simplification we consider that the distribution is linear. We consider $15 \%$ difference of concentration.

The comparison of the film mass with the film thickness allows us to draw the conclusion that Teflon in these films is porous, because films with the same fraction of gold and the same thickness have different mass. In support of this assumption an ellipsometry measurement was carried out. The comparison of the film mass with the film thickness allows us to draw the conclusion that Teflon in these films is porous, because films with the same fraction of gold and the same thickness have different mass. Ellipsometry measurements were carried out in support of this assumption.

Ellipsometers measure the ratio of complex amplitude reflection coefficients for the $p$ - and $s$ - polarization $R_{p} / R_{s}$ which is generally written in terms of two ellipsometrical angles $\Psi$ and $\Delta^{e l}[34]$.

$$
R_{p} / R_{s}=\tan \Psi_{\text {exp }}\left(j \Delta^{e l}\right)
$$

where $\tan \Psi=\left|R_{p} / R_{s}\right|$ represents the relative attenuation of the $p$ - and $s$-polarized components while $\Delta^{e l}$ represents the relative phase shift. Experimental values of the ellipsometrical angles $\Psi_{\exp }, \Delta_{\exp }^{e l}$ at various incidence angles $\varphi$ were obtained using an ellipsometer LEF-3M-1 equipped with He-Ne laser $(\lambda=632.8)$.

We will consider the Teflon film is the isotropic transparent layer [35,36]. The solution of reverse problem is to find such value of refractive index and layer thickness that provide the best match between the calculated $\Psi_{c a l}$, $\Delta_{c a l}^{e l}$ and the experimental $\Psi_{\text {exp }}, \Delta_{\text {exp }}^{e l}$ angular dependencies of $\Psi$ and $\Delta^{e l}$. This may be done by minimizing the object function [34]

$$
G=\sum_{i=1}^{N}\left\{\left(\Psi_{\text {exp }}\left(\varphi_{i}\right)-\Psi_{c a l}\left(\varphi_{i}\right)\right)^{2}+\left(\Delta_{\text {exp }}^{e l}\left(\varphi_{i}\right)-\Delta_{c a l}^{e l}\left(\varphi_{i}\right)\right)^{2}\right\}
$$

where $N$ is the number of incidence angles. The search of $G$ minimum was carried out using the modified NelderMead method [37].

The thin dense films of deposited Teflon have refractive index value $n=1.38$ [38]. Experimental values of $\Psi$ and $\Delta^{e l}$ for the $h_{A F M}=60 \mathrm{~nm}$ Teflon film are pre- sented in Figures 5(a) and (b), curve 4.

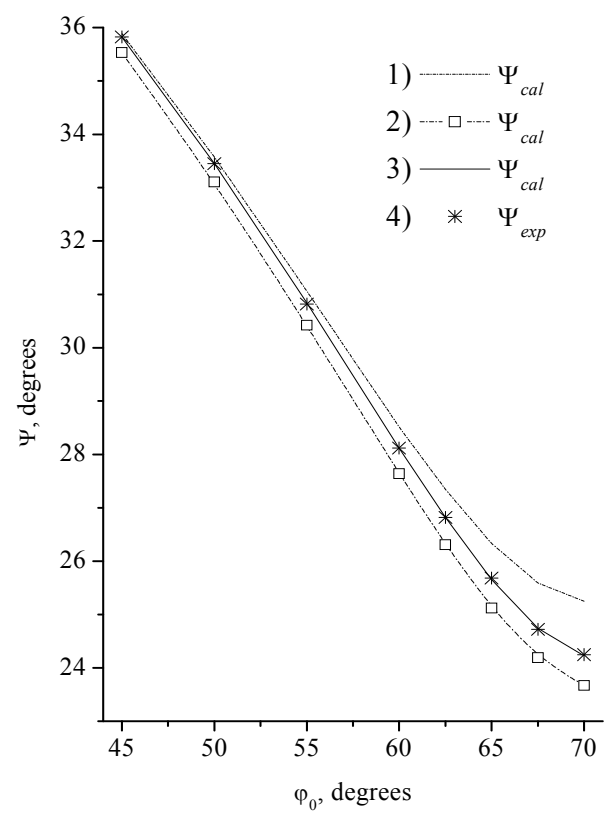

(a)

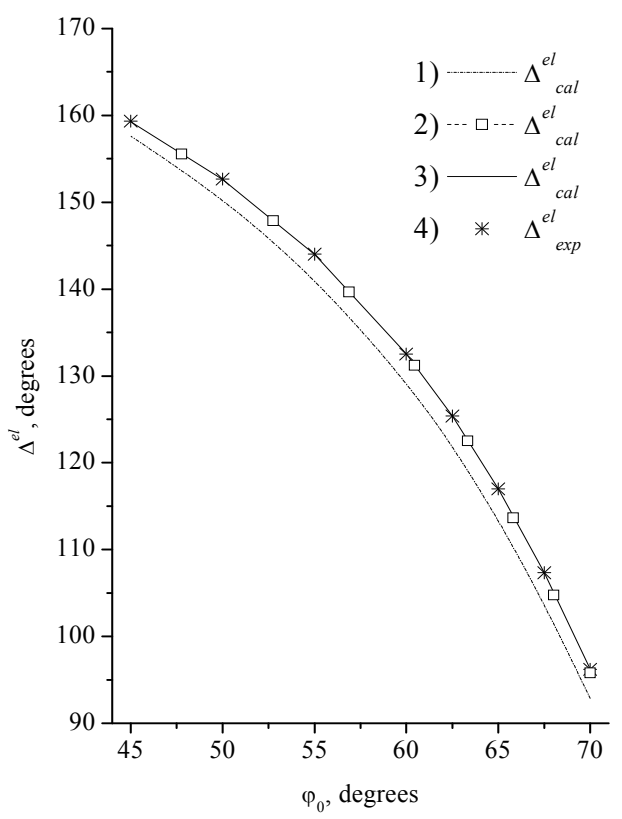

(b)

Figure 5. The angular dependences of a) $\Psi_{c a l}$ calculated at $n=1.38, h=60 \mathrm{~nm}$ (curve 1); $\Psi_{\text {cal }}$ calculated at $n=1.38, h$ $=54.45 \mathrm{~nm}$ (curve 2); $\Psi_{\text {cal }}$ calculated at $n=1.339, h=59.5$ nm (curve 3); experimental $\Psi_{\text {exp }}$ (curve 4). and b) $\Delta_{\text {cal }}^{e l}$ calculated at $n=1.38, h=60 \mathrm{~nm}$ (curve 1); $\Delta_{\text {cal }}^{e l}$ calculated at $n=1.38, h=54.45 \mathrm{~nm}$ (curve 2); $\Delta_{\text {cal }}^{e l}$ calculated at $n=$ 1.339, $h=59.5 \mathrm{~nm}$ (curve 3); experimental $\Delta_{\text {exp }}^{e l}$ (curve 4) 
Curves 1 in Figure 5 presents angular dependences of $\Psi_{c a l}, \quad \Delta_{c a l}^{e l}$ for the film with $n=1.38$ and $h=60 \mathrm{~nm}$. The considerable difference between the measured and calculated values of the ellipsometrical angles is observed in Figure 5. This difference may be caused by the inaccuracy in AFM-measurement and/or by a possible difference of the film's refractive index from value 1.38 . $\mathrm{Cu}$ rves 2 in Figure 5 presents the results of $G$-minimization corresponding to variation of the film thickness $(n=$ 1.38). The difference between experimental and calculated values of $\Psi, \Delta^{e l}$ is still rather considerable. It supposes a discrepancy between the refractive index value of the real film and $n=1.38$. At the same time, $G$-minimization due to variation of both $n$ and $h$ of the film (curves 3 in Figure 5) make it possible to reach the coincidence between measured and calculate values of $\Psi, \Delta^{e l}$, where $h=59.5 \mathrm{~nm}$ and $n=1.339$. The good coincidence between AFM- and ellipsometrical film's thickness takes place in this case.

Thus, during ellipsometrical investigations we found that refractive index values of Teflon films can vary in the range 1.17-1.34 in dependence of deposition regimes. Corresponding to this data, the Teflon in the film has 3$15 \%$ decrease in the index of refraction. Therefore, the volume concentration of gold in the films can be less than the specified value. We have used this fact in the modeling.

\section{Modeling}

Modeling of the optical properties of nano-composite films is usually done within the framework of the effective medium theory [39-42]. One should note that the effective medium theory operates with the linear response to a local (total) field. When the linear dimensions of the system under consideration are much greater than the characteristic dimensions of the local field inhomogeneity, one can describe the optical properties of the system by an effective dielectric function which defines the linear response to the total field. In the case of small particles (mesoparticles) or thin films, the characteristic dimensions of the local field inhomogeneity can be comparable to the linear dimensions of the particle (the thickness of the film). Then the local field effect can essentially influence the optical properties of the film. Indeed, it is well known that the local field distribution at small objects is very inhomogeneous and it can be observed by near-field scanning microscopy [43-45]. Taking into account the local field corrections becomes very important when the film is inhomogeneous along its thickness. These local-field corrections can be easily taken into account in the framework of linear response.

For exact consideration of the optical properties of small particles (or thin films) it is necessary to define the effective susceptibility as the response to an external fi- eld. Then the dielectric function will play the role of the characteristic of the material, and the linear response to the external field will play the role of the characteristic of the sample (a particle or a thin film). The idea of defining the linear response to an external field consists of the following:

For an unbounded medium (material), one can calculate by quantum mechanical methods the density of the local current caused by a local field. This current is related to the total field by a constitutive equation, which in the local approach is written as

$$
j_{i}(\vec{R}, \omega)=-i \omega \chi_{i j}(\vec{R}, \omega) E_{j}(\vec{R}, \omega)
$$

The mesoparticle is made of the same material and characterized by volume $V$ (or thin film with thickness $h$ ) and by the function of linear response $\chi_{i j}(\vec{R}, \omega)$. The external field $E_{i}^{(0)}(\vec{R}, \omega)$ acting on this object induces a local current at the point $\vec{R}$. It is clear that the local field $E_{i}(\vec{R}, \omega)$ in general does not equal the external field $E_{i}^{(0)}(\vec{R}, \omega)$. Besides that, it is possible that in the region near the surface (in case of the film, all film surface is near the surface region) this field strongly differs from $E_{i}^{(0)}(\vec{R}, \omega)$. And the current inside the object is proportional to an exterior field in the linear approach (that is when the exterior field is not rather strong). Then one can write

$$
j_{i}(\vec{R}, \omega)=-i \omega X_{i j}(\vec{R}, \omega) E_{j}^{(0)}(\vec{R}, \omega)
$$

where the current in each point of the film (particle) is related to an external field in this point (one should to note that the external field is controlled in an experiment). The tensor $X_{i j}(\vec{R}, \omega)$ is the effective susceptibility tensor. It depends both on the shape and the size of the object and determination of this tensor for the system is a main problem in linear response theory.

We now calculate the susceptibility of a nano-composite thin film for the case where the film is characterized by an inhomogeneous distribution of inclusions directed along the normal to the surface of the film. The film is located in space as follows: the XOY plane lies in the film plane and the $\mathrm{OZ}$ axis is perpendicular to it. Let us suppose that the initial susceptibility at any cross-section of the film (where $\Delta l$ is the thickness) can be characterized by its volume. The initial susceptibility $\chi(z)$ describes the inhomogeneous distribution of the inclusions along $\mathrm{OZ}$.

Then, taking into account that the film is homogeneous in the film plane, we can use the k-z-representation, which is a Fourier transform in the XOY plane. Obviously, the local current in the k-z-representation, $j(\vec{k}, z, \omega)$, strongly differs from the local current $j(\vec{R}, \omega)$. Because of these circumstances we introduce the local current in 
k-z-representation, $J(\vec{k}, z, \omega)$. As a result, the local (self-consistent) field $E_{i}(\vec{k}, z, \omega)$ has to obey the Lippmann-Schwinger Equation (21)

$$
\begin{aligned}
E_{i}(\vec{k}, z, \omega)= & E_{i}^{(0)}(\vec{k}, z, \omega) \\
& -a \int_{-h / 2}^{h / 2} d z^{\prime} G_{i j}\left(\vec{k}, z, z^{\prime}, \omega\right) \chi\left(z^{\prime}, \omega\right) E_{j}\left(\vec{k}, z^{\prime}, \omega\right)
\end{aligned}
$$

where $\chi(z, \omega)=(\varepsilon(z, \omega)-1), \varepsilon(z, \omega)$ is the dielectric volume function of the film material, $G_{i j}\left(\vec{k}, z, z^{\prime}, \omega\right)$ is the Green function (photon propagator) of the medium without the film and $a=\omega^{2} / c^{2}$, where $c$ is the speed of light. In our case we search for the response to a local field in contrast to the EMA [46,47]. At writing Equation (3), it was taken into account that the local current is connected with the local field via the constitutive equation

$$
J_{i}(\vec{k}, z, \omega)=-i \omega \chi(z, \omega) E_{i}(\vec{k}, z, \omega)
$$

On the other hand, the effective susceptibility $\mathrm{X}_{i j}(\vec{k}$, $z, \omega)$ is related to the local current and the external field $E_{j}^{(0)}(\vec{k}, z, \omega)$ as

$$
J_{i}(\vec{k}, z, \omega)=-i \omega \mathrm{X}_{i j}(\vec{k}, z, \omega) E_{j}^{(0)}(\vec{k}, z, \omega)
$$

Using this equation we can write the solution of Equation (3) in the general form

$$
\begin{aligned}
E_{i}(\vec{k}, z, \omega)= & E_{i}^{(0)}(\vec{k}, z, \omega)-a \int_{-h / 2}^{h / 2} d z^{\prime} G_{i j}\left(\vec{k}, z, z^{\prime}, \omega\right) . \\
& \mathrm{X}_{j l}\left(\vec{k}, z^{\prime}, \omega\right) E_{l}^{(0)}\left(\vec{k}, z^{\prime}, \omega\right)
\end{aligned} .
$$

The connection with local and external fields can easily be obtained from Equations (6) and (7)

$$
E_{i}(\vec{k}, z, \omega)=(\chi(z, \omega))^{-1} \mathrm{X}_{i j}(\vec{k}, z, \omega) E_{j}^{(0)}(\vec{k}, z, \omega) .
$$

The effective susceptibility takes the form [48]

$$
\mathrm{X}_{j l}(\vec{k}, z, \omega)=\chi(z, \omega) \Omega_{j l}(\vec{k}, z, \omega),
$$

where the factor

$$
\Omega_{j l}(\vec{k}, z, \omega)=\left[\delta_{l j}+a \int_{-h / 2}^{h / 2} d z^{\prime} G_{l j}\left(\vec{k}, z^{\prime}, z, \omega\right) \chi(z, \omega)\right]^{-1},
$$

represents the local field correction. We note that the selfenergy part

$$
S_{l j}(\vec{k}, z)=a \int_{-h / 2}^{h / 2} d z^{\prime} G_{l j}\left(\vec{k}, z^{\prime}, z\right) \chi(z, \omega),
$$

describes the influence of the nonlocal electrodynamical interaction inside the film. The calculated effective susceptibility is the necessary characteristic for the definition of the absorption spectrum of such a system.
In order to calculate the absorbed energy from the external field by the film, we need the dissipative function, which has the interpretation of the energy absorbed per unit volume of the film per unit of time:

$$
Q=\frac{1}{4}<\overline{\left(J_{i}+J_{i}^{*}\right)\left(E_{i}+E_{i}^{*}\right)}>.
$$

Here, $J_{i}(\vec{R})$ is the local current inside the film, $E_{i}$ $(\vec{R})$ is the local field, and $\overline{(\ldots)}$ and $<\ldots>$ indicate the average over time (much longer than the period of the external field) and volume, respectively.

Because the local current can be expressed in terms of the external field, Equation (7), and the local field is connected with the external field as in Equation (9), Equation (13) can be rewritten in the form

$$
Q=2 \omega I_{0}<\operatorname{Im} \chi(z, \omega) \Omega_{i}(\vec{k}, z, \omega)\left(\Omega_{j}(\vec{k}, z, \omega)\right)^{*}>e_{i} \cdot e_{j} .
$$

Here, $I_{0}=\left|E^{(0)}\right|^{2}$ is the intensity of the incident radiation, and $\vec{e}$ is the polarization vector of the incident field. Supposing that the absorbed radiation is linearly polarized (along $i$-th axes), one can rewrite Equation (12) in the form

$$
Q=2 \omega I_{0} \frac{1}{h} \int_{-h / 2}^{h / 2} d z \operatorname{Im}(\chi(z, \omega))\left|\Omega_{i i}(\vec{k}, z, \omega)\right|^{2} .
$$

Thus, having calculated the absorption of the simulateed system using Equations (13) and (9), and using the Green's function of two semi-infinite spaces with a flat interface [49], it is possible to compare the results with the experimental spectra of the real film. The distribution function for the shape can be taken as the distribution which forms the composite. It will be a Gaussian distribution of particles. During the calculation we noticed that the width of the distribution plays a major role [45] (not the exact form of the distribution). For simplification of the analytical result we take a step-like distribution. For ellipsoidal particles we have the $L_{x}=L_{y}$ for the depolarization factors. The simplest form of the distribution function $P(L)$ that deserves attention in this case is the oneparameter step-like distribution [[51]].

$$
P(L)=\frac{1}{\Delta} \vartheta\left(L-\frac{1}{3}+\frac{1}{3} \Delta\right) \vartheta\left(-L+\frac{1}{3}+\frac{2}{3} \Delta\right) .
$$

where $\vartheta(\ldots)$ is the Heaviside step function and $\Delta$ is a parameter signifying a width of the nonspherisity distribution of the particles. This distribution rather precisely corresponds to the distribution of the particles in the film produced by our method. Also, for real samples it is necessary to take into account that the particles are in some sort of shell [50]. 


\section{Results and Discussion}

We now present some results calculated with the help of the modeling method described above. We shall compare the results of the calculations with the experimental results. The main purpose of this comparison is the definition of the structure of the nano-composite film. But the first step of the calculation is the verification of the adequacy of the proposed model. To demonstrate the localfield effects (the influence of the thickness of the film and its structure on the absorption profile), we first consider a system consisting of a thin polymer film (Teflon) with a dielectric constant a little below the literature value, because of the porous structure of our film [31]. The optical constants for Au were taking from [32]. Then we calculated the absorption profile of the gold-Teflon nanocomposite film and compared the model to the experimental results.

The calculated dependence of the absorption profile of the film, with a thickness of $100 \mathrm{~nm}$, on the concentrateion of gold particles is shown in Figure 6. The absorption profile becomes more intensive, more extended and slightly shifted to short wavelengths when the concentration of inclusions grows. Monotonous increasing of the concentration leads to a non-monotone change of the absorption profile. This is a nontrivial fact. Namely, the increase of the concentration of inclusion particles leads to a strong variation of the local field at the sites of the particles. Then, the absorption processes by the inclusion particles occurs under a different condition. As a result, this leads to a change of the absorption profile.

The dependence of the absorption profile on the width of the shape distribution $\Delta$ is presented in Figure 7. We see that increasing the width of the distribution leads to a shift of the long-wavelength front of the absorption pro file towards the red. In the same time the half-width of

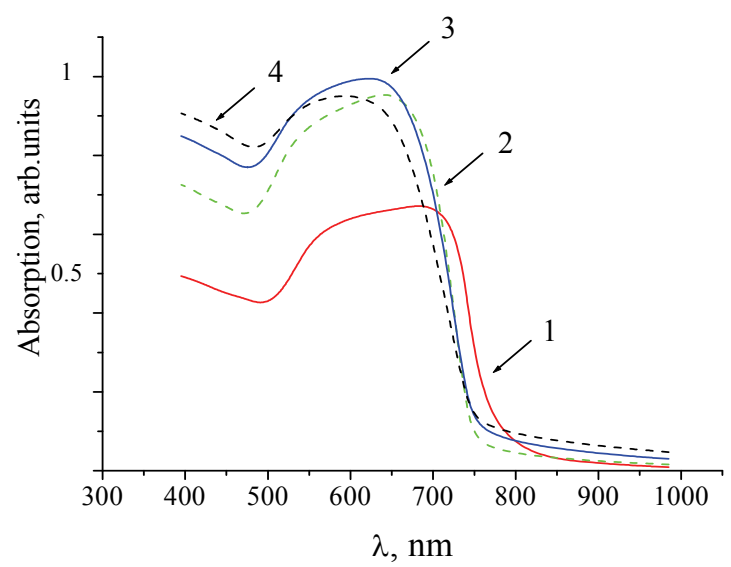

Figure 6. Calculated absorption spectra of the $100 \mathrm{~nm}$ AuTeflon composite film with different parts of Au inclusions: 1) $10 \%$; 2) $20 \%$; 3) $30 \%$; 4) $40 \%$. Particles in the film have mainly a spherical form with the form distribution $\Delta=0.5$ the profile is proportionally broadened when $\Delta$ increases. And the absorption profile changes to a "two-peak" line. The shape distribution centre, which is defined by the depolarization factor [50,51], influences the peak position of the absorption profile. The effect of the shape of the inclusion particles on the absorption profile is shown in Figure 8. In Figure 8, curve 3, the absorption profile for the composite film in which the inclusions are distributed around spherical particles (depolarization factor $L=0.3$ ) with $\Delta=0.5$ is shown. We see that the peak at $550 \mathrm{~nm}$ corresponds to spherical particles. Curve 4 in Figure 8 shows that mainly oblate ellipsoidal particles in the composite lead to a narrowing of the absorption profile, and the peak shifts to the blue. On the contrary, the composite film with mainly prolate ellipsoidal inclusions (Figure 8, curves 1 and 2 ) is characterized by a slightly broadened absorption profile with the peak shifted to red.

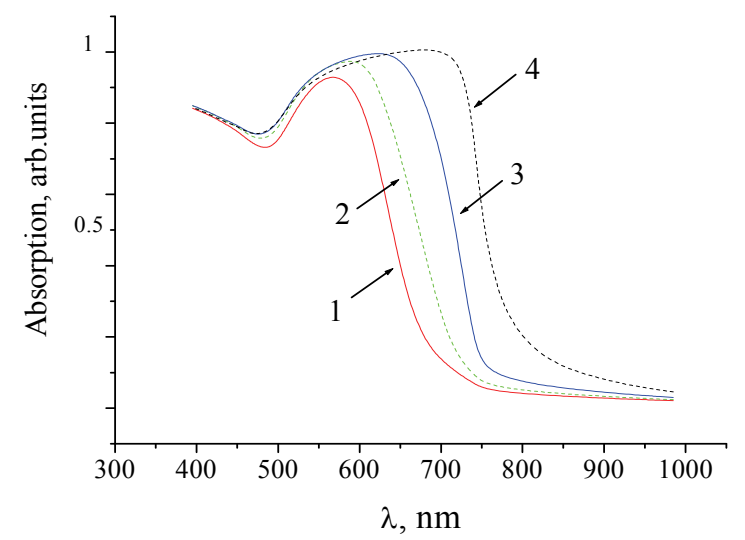

Figure 7. Calculated absorption spectra of the $100 \mathrm{~nm}$ Au-Teflon composite film with different widths of the form distribution $\Delta: 1) 0.3$; 2) 0.4 ; 3) 0.5 ; 4) 0.6 . Particles in the film have mainly a spherical form and the Au mass concentration is $\mathbf{3 0 \%}$

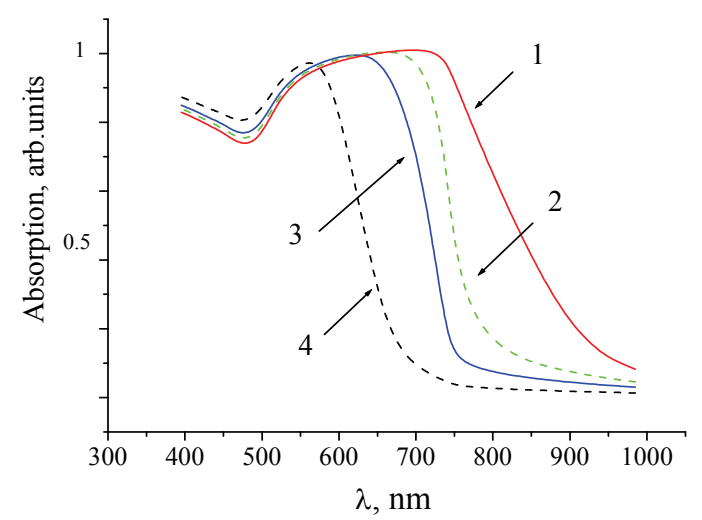

Figure 8. Calculated absorption spectra of the $100 \mathrm{~nm}$ Au-Teflon composite film with different depolarization factors: 1$) 0.2$; 2) 0.25 ; 3) 0.3 ; 4) 0.35 . Gold mass concentration is $30 \%$, form distribution is 0.5 
Presumably, during the vacuum evaporation Teflon forms holes which may be filled with gold. Thus, such formation looks like gold particles with an air shell in a Teflon environment (previous calculations do not consider a shell). The absorption of clear gold particles without a shell is shown in Figure 9 for shell-factor $p=0.99(p=$ 0 when a shell fills a volume of particles, $p=1$ when a shell is absent). The peak of the spectrum sharply shifts to the blue with the appearance of an air shell. Further increasing of the shell leads to reduction of the peak intensity. This happens due to the decrease of the gold fraction in the $\mathrm{Au} /$ Teflon film.

Combining these parameters can predict the optical properties of the thin Au-Teflon films, and give information about the structure and the form of the particles in the film.

As it can be seen from Figure 4, the absorption spectra are changed noticeably during the deposition of the film. We can compare this dependence on film growth with the calculated absorption spectra. The dependence on the thickness of the film has the same behavior at 500-600 $\mathrm{nm}$, at the peak of the graph. Also we see that the width of the distribution increases with time of growth. We suppose that the shape of the Au particles varies during the deposition process from oblate ellipsoids to spherical particles. Indeed the long-wavelength part of the spectra is characterized by these changes of the particle shape. We explain the dip of the curve at $410-420 \mathrm{~nm}$ as due to imperfections of our instruments at near-ultraviolet wavelengths.

In Figure 10 the absorption spectra of $\mathrm{Au} / \mathrm{Teflon}$ with $2 \% \mathrm{Au}$ are shown. The intensity of absorption is much lower in this case, and is explained by the low concentration of gold, and this corresponds to the theoretical calculations (Figure 9). In the model, it is possible to say that

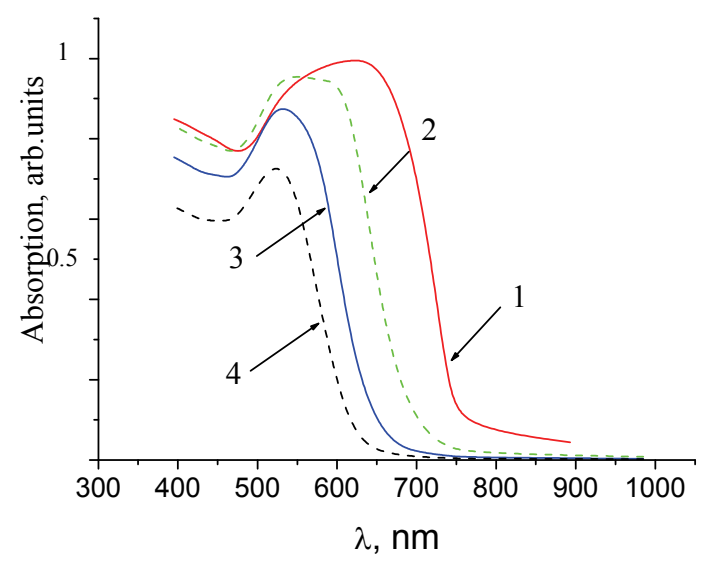

Figure 9. Calculated absorption spectra of the $100 \mathrm{~nm}$ Au-Teflon composite film were the gold mass concentration is $30 \%$, the depolarization factor is 0.3 and the form distribution is 0.5 . Profiles have different shell-factor: 1) 0.99 ; 2) 0.7 ; 0.5 ; 4) 0.3 the particles in this sample are characterized by smaller sizes with a smaller width of the form distribution. In case of $2 \% \mathrm{Au}$ concentration, it can be calculated that our model is in $\sim 10 \%$ accuracy with experimental results in the interested wavelength range.

The absorption spectra of Au-3 and Au-2 (Figure 11) samples completely coincide with the calculated spectra. In our model it was assumed that sample 3 has a narrow form distribution $(\sim 0.1)$. The form of the spectra differs due to the change of the form factor, where for the Au-2 sample there are more extended particles (depolarization factor of 0.25 ). Also the form is different because of the

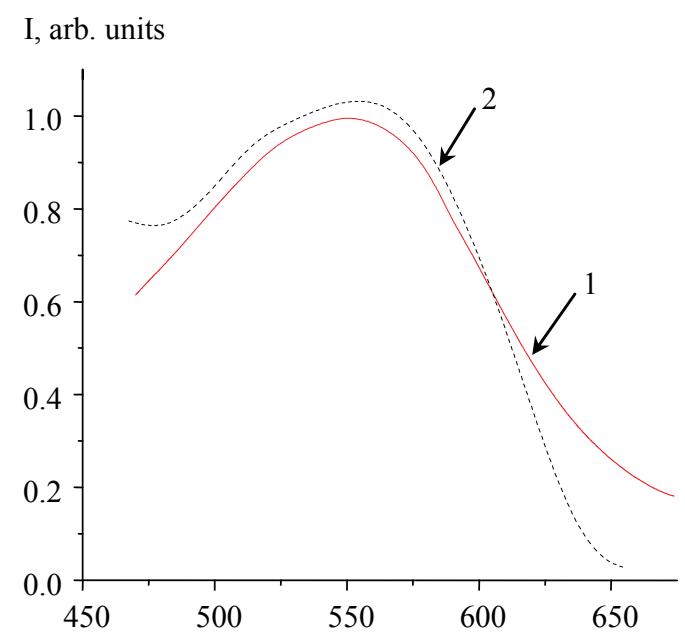

Figure 10. Absorption spectra for $2 \%$ Au volume filling (Au-3). 1) Experimental absorption spectra, 2) Calculated absorption spectra for $2 \%$ Au filling, $1.4 \mathrm{~nm}$ diameter of mainly spherical inclusions with $\Delta=0.1$, thickness $27 \mathrm{~nm}$

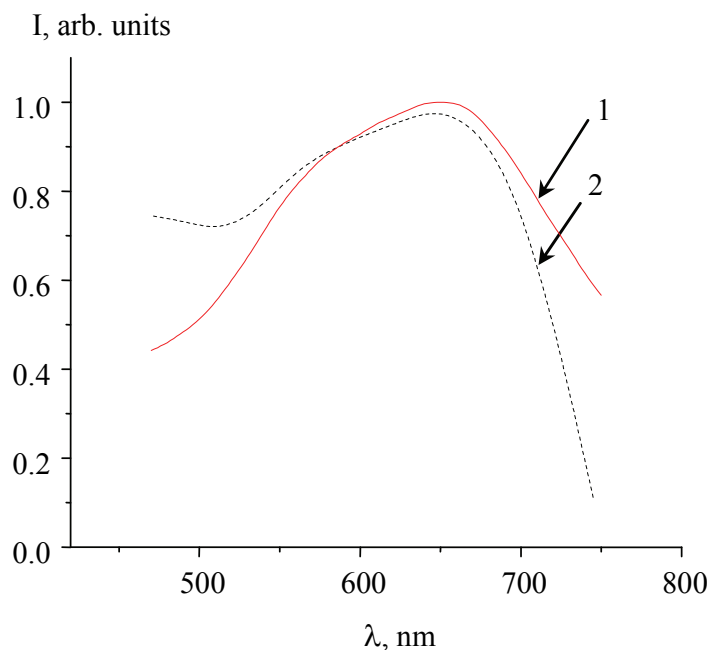

Figure 11. Absorption spectra for $13 \%$ Au volume filling (Au-2). 1) Experimental absorption spectra, 2) calculated absorption spectra for $13 \%$ Au filling, $4-7 \mathrm{~nm}$ diameter of prolate $(\mathrm{L}=\mathbf{0 . 2 5})$ inclusions with $\Delta=\mathbf{0 . 3}$ 


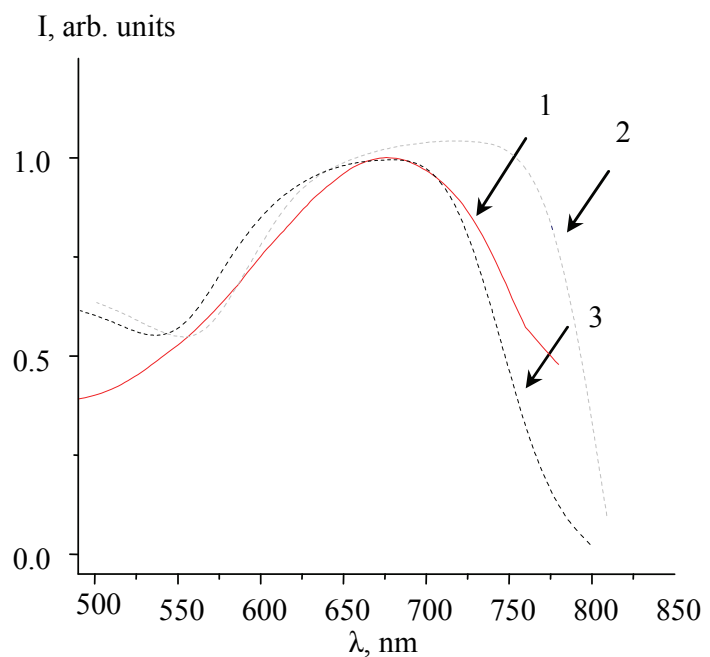

Figure 12. Absorption spectra for $35 \%$ Au volume filling (Au-1). 1) Experimental absorption spectra; 2) calculated absorption spectra for $21 \%$ Au filling, $2.5-5 \mathrm{~nm}$ diameter of spherical inclusions with $\Delta=0.5,3) 35 \%$ Au filling, 2.5-5 $\mathrm{nm}$ diameter of spherical inclusions with $\Delta=0.5, \mathrm{p}=0.86$

increase in concentration and the increase of delta. At $550 \mathrm{~nm}(\mathrm{Au}-2)$ we see an additional peak, which is not observed in the Au-1 experimental spectra (Figure 12, curve 1). Theoretical spectra also display this additional peak, and consequently we can assume that for this concentration and thickness of film we find a combined structure. A fraction of the gold can consist of sticks which have grown through the thickness of the film and have become common spherical particles. Comparing the spectra for sample 1, it has been established that the volume concentration of gold decreased to $21 \%$. This can be explained by the occurring porous structure in Teflon. Indeed, measurements of film mass, mass of inclusions and film thickness allow us to make the conclusion that in the Teflon matrix the low density domains appear. These "air pores" have an influence on the film parameters and, obviously, on the absorption profiles. This can be taken into account in two ways in the model. We can suppose that Teflon has less density, and therefore the volume concentration of inclusions effectively decreases (Figure 12, curve 2). On the other hand we can assume that the inclusions become surrounded by a low density shell (the gold in air system arises). Then we should consider the nano-composite with shelled particles. The calculations for an inclusions concentration of $35 \%$, and an air shell with $p=0.86$, were taken into account. The results of this modeling are shown in Figure 12 (curve 3). We see that the shelling model is more applicable to the experimental situation.

\section{Conclusions}

The thermal vacuum deposition method was used for ob- taining three-component composite thin Au-Teflon films of different concentrations of inclusions. The structure of the obtained films was studied using TEM and AFM. It was established with Fourier analysis of the TEM images that inclusion particles have a mainly near-spherical shape. The method for numerical simulations of the optical properties of nano-composite films was developed. The method is based on the effective susceptibility concept. Both two-component and three-component (Au particles with air shell) nano-composite optical properties could be calculated. The absorption profiles of nano-composite Au-Teflon films were calculated. The dependence of the absorption profiles on the concentration and shape was analyzed. It was found that both the concentration and the shape of the inclusions strongly influence the absorption processes in the films. We found that varying the shape of the particles from oblate to prolate ellipsoids leads to deformation and a red shift of the absorption profile. Vacuum deposited Au-Teflon films were studied experimentally. Particularly, the absorption profiles of the films were measured. Comparison of the experimental and theoretical absorption spectra allows us to draw conclusions about the concentration, the shape of the particles and the shape distribution of the inclusions. It was established that in the case of thin films with low concentrations (Au-3 sample) of inclusions the shape of the inclusions is mainly spherical. When the film beco- mes thicker and the inclusions concentration increases, the shape of the inclusions becomes mainly a prolate ellipsoid. Our approach can be useful for further developpments of technological methods for obtaining two- and three-component nano-composite films.

\section{Acknowledgements}

This work was supported, in part, by Grant No 3480 awarded by the Science \& Technology Center in Ukraine (STCU).

\section{REFERENCES}

[1] L. Jacak, P. Hawrylak and A. Wojs, "Quantum Dots," Springer, Berlin, 1998.

[2] S. Stagira, M. Nisoli, S. De Silvestri, A. Stella, P. Tognini, P. Cheyssac and R. Kofman, "Ultrafast Optical Relaxation Dynamics in Metallic Nanoparticles: From BulkLike toward Spatial Confinement Regime," Chemical Physics, Vol. 251, No. 1-3, 2000, pp. 259-267.

[3] R. Kofman, P. Cheyssac, A. Aouaj, Y. Lereah, G. Deutscher, T. Ben David, J. M. Penisson and A. Bourret, "Surface Melting Enhanced by Curvature Effects," Surface Science, Vol. 303, No. 1-2, 1994, pp. 231-246.

[4] F. Ercolessi, W. Andreoni and E. Tosatti, "Melting of Small Gold Particles: Mechanism and Size Effects," Physics Review Letters, Vol. 66, No. 7, 1991, pp. 911-914.

[5] N. Cioffi, I. Farella, L. Torsi, A. Valentini and A. Tafuri, "Correlation between Surface Chemical Composition and 
Vapor Sensing Properties of Gold-Fluorocarbon Nanocomposites," Sensors and Actuators B, Vol. 84, No. 1, 2002, pp. 49-54.

[6] A. V. Goncharenko, D. O. Grynko, K. P. Grytsenko and V. Z. Lozovski, "Preparation and Optical Properties of $\mathrm{Au} /$ Teflon Nanocomposites," Journal of Nanoscience and Nanotechnology, Vol. 5, No. 11, 2005, pp. 1919-1924.

[7] J. H. Holts and S. A. Asher, "Polymerized Colloidal Crystal Hydrogel Films as Intelligent Chemical Sensing Materials," Nature, Vol. 389, 1997, pp. 829-832.

[8] A. D'Addabbo, A. Valentini and A. Convertin, "Swelling of CFx and $\mathrm{CFx}(\mathrm{Au})$ Films," Journal of Applied Physics, Vol. 87, No. 1, 2000, pp. 2039-2043.

[9] A. Convertino, A. Capobianchi, A. Valentini and E. N. Cirillo, "A New Approach to Organic Solvent Detection: High-Reflectivity Bragg Reflectors Based on a Gold Nanoparticle/Teflon-like Composite Material," Advanced Materials, Vol. 15, No. 13, 2000, pp. 1103-1105.

[10] S. I. Bozhevolnyi, V. S. Volkov and K. Leosson, "Localization and Waveguiding of Surface Plasmon Polaritons in Random Nanostructures," Physics Review Letters, Vol. 89, No. 18, 2002, pp. 186801-186805.

[11] I. V. Barca, A. P. Brown, M. P. Andrews, T. Galstian, et al., "Linear and Nonlinear Optical Response of Dye Anchored to Gold Nanoparticles," Canadian Journal of Chemistry, Vol. 80, 2002, pp. 1625-1633.

[12] K. G. Thomas and P. Kamat, "Chromophore-Functionalized Gold Nanoparticles," Accounts of Chemical Research, Vol. 36, No. 12, 2003, pp. 888-898.

[13] Y. P. Bao, M. Huber, T.-F. Wei, S. S. Marla, J. J. Storhoff and U. R. Müller, "SNP Identification in Unamplified Human Genomic DNA with Gold Nanoparticle Probes," Nucleic Acids Research, Vol. 33, No. 2, 2005, p. e15.

[14] H. Hakkinen, S. Abbet, A. Sanchez, U. Heiz and U. Landmanet, "Structural, Electronic, and Impurity-Doping Effects in Nanoscale Chemistry: Supported Gold Nanoclusters," Angewandte Chemie International Edition, Vol. 42, No. 11, 2003, pp. 1297-1300.

[15] A. Biswas, O. C. Aktas, J. Kanzow, U. Saeed, T. Strunskus, V. Zaporojtchenko and F. Faupel, "Polymer-Metal Optical Nanocomposites with Tunable Particle Plasmon Resonance Prepared by Vapor Phase Co-Deposition," Materials Letters, Vol. 58, No. 9, 2004, pp. 1530-1534.

[16] U. Schurmann, W. Hartung, H. Takele, V. Zaporojtchenko and F. Faupel, "Controlled Syntheses of Ag-Polytetrafluoroethylene Nanocomposite Thin Films by Co-Sputtering from Two Magnetron Sources," Nanotechnology, Vol. 16, No. 8, 2005, p. 1078.

[17] A. Kiesow, J. E. Morris, C. Radehaus and A. Heilmann, "Switching Behavior of Plasma Polymer Films Containing Silver Nanoparticles," Journal of Applied Physics, Vol. 94, No. 10, 2003, pp. 6988-6991.

[18] E. Dulkeith, T. Niedereichholz, T. A. Klar, J. Feldmann, G. von Plessen, D. I. Gittins, K. S. Mayya and F. Caruso, "Plasmon Emission in Photoexcited Gold Nanoparticles," Physical Review B, Vol. 70, No. 20, 2004, pp. 205424205428.
[19] Y. Yang, J. Huang, S. Liu and J. Shen, "Preparation, Characterization and Electroluminescence of Zns Nanocrystals in a Polymer Matrix," Journal of Material Chemistry, Vol. 7, No. 1, 1997, pp. 131-133.

[20] L. A. Baraban and V. Z. Lozovski, "Reflection and Absorption of Light by a Thin Semiconductor Film," Optics and Spectroscopy, Vol. 97, No. 5, 2004, pp. 810-816.

[21] O. Keller, "Local Fields in the Electrodynamics of Mesoscopic Media," Physics Reports, Vol. 85, No. 2-3, 1996, pp. 85-262.

[22] J.-J. Griffet and R. Carminati, "Image Formation in Near-Field Optics," Progress in Surface Science, Vol. 56, No. 3, 1997, pp. 133-237.

[23] "Near Field Optics," In: D. W. Pohl and D. Courjon, Eds., Kluwer, Dordrecht, 1993.

[24] S. I. Bozhevolnyi, "Optics of Nanostructured Materials," In: V. M. Markel and T. F. George, Eds., Wiley, New York, 2001.

[25] C. Girard, C. Joachim, and S. Gauthier, "The Physics of the Near-Field," Reports on Progress in Physics, Vol. 63, No. 6, 2000, pp. 893-938.

[26] L. A. Cury, L. O. Ladeira and A. Righi, "Large Blue Shift in the Absorption Spectra of BEH-PPV Films Containing Gold Nanoparticles," Synthetic Metals, Vol. 139, No. 2, 2003, pp. 283-286.

[27] H. Song, O. J. Ilegbusi and L. I. Trakhtenberg, "Modeling vapor Deposition of Metal/Semiconductor-Polymer Nanocomposite," Thin Solid Films, Vol. 476, No. 1, 2005, pp. 190-195.

[28] G. Carotenuto and L. Nicolais, "Synthesis and Characterization of Gold-Based Nanoscopic Additives for Polymers," Composites, Part B, Vol. 35, No. 5, 2004, pp. 385 391.

[29] D. Gritsenko, V. Grynko, V. Lozovski, J. Friedrich, R.-D. Schulze, J. Jurga, A. Convertino and A. Kotko, "Properties of Au-Nano-Clusters in PTFE Film Prepared by Co-Evaporation in a Vacuum," New Directions in Modification and Application of Polymers, Wydanictwo Politechniki Poznanskiej, Poland, 2004.

[30] C. K. O'Sullivan and G. G. Guilbault, "Commercial Quartz Crystal Microbalances-Theory and Applications," Biosensors and Bioelectronics, Vol. 14, No. 8-9, 1999, pp. 663-670.

[31] J. C. Salamone, "Polymeric Materials Encyclopedia" CRC Press, New York, 1996.

[32] P. B. Johnson and R. W. Christy, "Optical Constants of the Noble Metals," Physical Review B, Vol. 6, No. 12, 1972, pp. 4370-4379.

[33] H. Stark, "Applications of Optical Fourier Transforms," Radio i Svyaz’, Moscow, 1988.

[34] R. M. Azzam and N. M. Bashara, "Ellipsometry and Polarized Light," Holland Publishing, Amsterdam, 1977.

[35] M. V. Sopinskyy, P. E. Shepelyavyi, A. V. Stronski and E. F. Venger, "Ellipsometry and AFM Study of Post- Deposition Transformations in Vacuum-Evaporated As-S-Se Films," Journal of Optoelectronics and Advanced Mate- 
rials, Vol. 7, No. 5, 2005, pp. 2255- 2266.

[36] I. V. Lysy, O. I. Vlasenko, M. V. Sopinskyy, A. O. Gubanova and T. A. Kryskov, "Ellipsometric Measurements of Refractive Index of Chalcogenide and ChalcogenideBased Bulk Glassy Samples," Moldavian Journal of the Physical Sciences, Vol. 6, No. 2-3, 2007, pp. 320-328.

[37] J. A. Nelder and R. Mead, "A Simplex Method for Function Minimization," Computational Journal, Vol. 7, No. 2, 1965, pp. 308-313.

[38] M. Pierno, C. S. Casari, R. Piazza and C. E. Bottani, "Structural Evolution of Crystalline Polymer Latex Films: Propagating and Confined Acoustic Modes," Applied Physics Letter, Vol. 82, No. 10, 2003, pp. 1532-1535.

[39] D. J. Bergman, "The Dielectric Constant of a Composite Material-A Problem in Classical Physics," Physics Reports, Vol. 43, 1978, pp. 377-407.

[40] D. J. Bergman and D. Stroud. "Physical Properties of Macroscopically Inhomogeneous Media," Solid-State Physics, Vol. 46, 1992, pp. 147-269.

[41] K. E. Peiponen, E. M. Varianen, J. J. Saarinen and M. O. A. Makinen, "The Dispersion Theory of Optically Linear and Nonlinear Bruggeman Liquids," Optics Communications, Vol. 205, No. 1-3, 2002, pp. 17-24.

[42] E. F. Venger, A. V. Goncharenko and M. L. Dmitruk, "Optics of Small Particles and Disperse Media," Naukova Dumka, Kyiv, 1999.

[43] D. W. Pohl, "Scanning Near-Field Optical Microscopy," In: C. J. R. Sheppard and T. Mulvey, Eds., Advances in Optical and Electron Microscopy, 12 Edition, Academic Press, London, 1990, p. 243.

[44] G. Kaupp, “Atomic Force Microscopy, Scanning Nearfield
Optical Microscopy and Nanoscratching: Application to Rough and Natural Surfaces," Springer, Heidelberg, 2006.

[45] V. Lozovski, Y. Nazarok and S. I. Bozhevolnyi, "NearField Imaging of Pyramid-Like Nanoparticles at a Surface," Physica E, Vol. 11, No. 4, 2001, pp. 323-331.

[46] V. Kochergin, V. Zaporojtchenko, H. Takele, F. Faupel, and H. Föll, "Improved Effective Medium Approach: Application to Metal Nanocomposites," Journal of Applied Physics, Vol. 101, No. 2, 2007, pp. 024302-024309.

[47] D. Dalacu and L. Martinu, "Spectroellipsometric Characterization of Plasma-Deposited Au/Fluoropolymer Nanocomposite Films," Journal Vacuum Science and Technology A, Vol. 17, No. 3, 1999, pp. 877-883.

[48] L. Baraban and V. Lozovski, "Light Absorption by Thin Nano-Composite Films with Different Distributions of Inclusions along Film Thickness," Semiconductor Physics, Quantum Electronics and Optoelectronics, Vol. 6, No. 3, 2009, pp. 667-672.

[49] A. A. Maradudin and D. L. Mills, "Scattering and Absorption of Electromagnetic Radiation by a Semi-Infinite Medium in the Presence of Surface Roughness," Physical Review B, Vol. 11, No. 4, 1975, pp. 1392-1415.

[50] A. V. Goncharenko, "Effective Dielectric Response of a Shape-Distributed Particle System," Journal of Physics: Condensed Matter, Vol. 13, No. 35, 2001, pp. 8217-8225.

[51] A. V. Goncharenko, "Generalizations of the Bruggeman Equation and a Concept of Shape-Distributed Particle Composites," Physical Review E, Vol. 68, No. 4, 2003, pp. 041108-041121. 